\title{
ON THE COHOMOLOGY OF SPLIT EXTENSIONS
}

\author{
D. J. BENSON AND M. FESHBACH
}

(Communicated by Thomas Goodwillie)

\begin{abstract}
We construct, for each value of $n$, a split extension of finite 2groups, with complement isomorphic to $Z / 2$, for which the differential $d_{n}$ is nonzero in the Lyndon-Hochschild-Serre spectral sequence.
\end{abstract}

\section{INTRODUCTION}

In general, the differentials in the Lyndon-Hochschild-Serre spectral sequence for a group extension are difficult to understand. In the case of a central extension, the $E_{2}$ page is easy to calculate and the transgressions may be computed using the fact that they commute with the Steenrod operations on the base and fiber. Internal differentials, however, are more difficult.

At the other extreme, in the case of a split extension with nontrivial action, even the $E_{2}$ page is hard to calculate. But it appears possible, from the calculations which have been achieved so far, that in the case of a split extension with abelian normal subgroup one might always have $E_{3}=E_{\infty}$. In case the normal subgroup is a torus (direct product of copies of the circle group $S^{1}$; in this case it is not really a Lyndon-Hochschild-Serre spectral sequence, but rather the Serre spectral sequence for the corresponding fibration of classifying spaces), the cohomology is concentrated in even degrees, so this would imply that $E_{2}=E_{\infty}$. So the restriction map in this case would be surjective onto the invariant elements of the cohomology of the torus (see [3] for some evidence for this). It also appears possible that if the normal subgroup is an elementary abelian 2-group and the coefficients are taken to be a field of characteristic two, then $E_{2}$ might again always equal $E_{\infty}$.

Our contribution in this paper is to show that in the above speculations the hypothesis that the normal subgroup is abelian cannot be dropped. Namely, we construct, for each value of $n$, a split extension of finite 2-groups, with complement isomorphic to $\mathrm{Z} / 2$, for which the differential $d_{n}$ is nonzero. The groups involved are nilpotent of class two, and the coefficients are taken to be a field of characteristic two. The example is a modification of an example constructed by Avrunin and Carlson [1] to show that the cohomology of a finite

Received by the editors May 12, 1992 and, in revised form, October 27, 1992.

1991 Mathematics Subject Classification. Primary 20 J06. 
group can have nilpotent elements with arbitrarily large degree of nilpotence (multiplicative exponent).

\section{EXTENSIONS WITH $\mathrm{Z} / 2$ QUOTIENT}

Suppose that $N \triangleleft G$ is a subgroup of index two, and let $g$ be an element of $G$ not in $N$. Then the extension $1 \rightarrow N \rightarrow G \rightarrow \mathbf{Z} / 2 \rightarrow 1$ corresponds to an element $x \in H^{1}\left(G, \mathbf{F}_{2}\right)$.

Lemma 2.1. The above extension splits if and only if $x$ is not nilpotent.

Proof. If the extension splits then every power of $x$ restricts to a nonzero cohomology class on the complement, so $x$ is not nilpotent. Conversely, if $x$ is not nilpotent then by a theorem of Quillen [4], $x$ has a nonzero restriction to some elementary abelian 2-subgroup, and since it has degree one, it therefore has a nonzero restriction to a cyclic subgroup of order two. This subgroup cannot be contained in $N$ because the restriction of $x$ to $N$ is zero.

Let $k$ be a field of characteristic two. Then we have a short exact sequence of $k G$-modules

$$
0 \rightarrow k \rightarrow k_{N} \uparrow^{G} \rightarrow k \rightarrow 0
$$

and hence a long exact sequence in cohomology

$$
\cdots \stackrel{x}{\rightarrow} H^{n}(G, k) \stackrel{\operatorname{res}_{G, N}}{\longrightarrow} H^{n}(N, k) \stackrel{\operatorname{Tr}_{N, G}}{\longrightarrow} H^{n}(G, k) \stackrel{x}{\rightarrow} H^{n+1}(G, k) \stackrel{\operatorname{res}_{G, N}}{\longrightarrow} \cdots .
$$

Here we have used the Eckmann-Shapiro isomorphism to identify $H^{*}\left(G, k_{N} \uparrow^{G}\right)$ with $H^{*}(N, k)$. The identification of the maps in the above sequence is an easy exercise in diagram chasing. Note that $\operatorname{res}_{G, N} \circ \operatorname{Tr}_{N, G}$ is equal to the action of $1+g$ on $H^{*}(N, k)$.

The above long exact sequence is related to the spectral sequence for the group extension as follows. Let $P_{*}$ be the minimal resolution of $k$ as a $k(\mathbf{Z} / 2)$ module so that each $P_{p}$ is isomorphic to $k(\mathrm{Z} / 2)$, and let $Q_{*}$ be a resolution of $k$ as a $k G$-module. Then the spectral sequence of the group extension may be taken to be the spectral sequence of the double complex

$E_{0}^{p q}=\operatorname{Hom}_{k G / N}\left(P_{p}, \operatorname{Hom}_{k N}\left(Q_{q}, k\right)\right) \cong \operatorname{Hom}_{k G}\left(P_{p} \otimes Q_{q}, k\right) \cong \operatorname{Hom}_{k N}\left(Q_{q}, k\right)$.

In this spectral sequence, we have

$$
D_{1}^{p q} \cong D_{1}^{0 q} \cong H^{q}\left(\operatorname{Tot}\left(E_{0}\right), d_{0}+d_{1}\right) \cong H^{q}(G, k)
$$

(see, for example, [2, pp. 110, 113]). We also have

$$
E_{1}^{p q}=\operatorname{Hom}_{k G / N}\left(P_{p}, H^{q}(N, k)\right) \cong H^{q}(N, k)
$$

so that $E_{1}^{* *} \cong k[x] \otimes H^{*}(N, k)$, with product given by

$$
\left(x^{i} \otimes a\right)\left(x^{j} \otimes b\right)=x^{i+j} \otimes\left(g^{j}\right)^{*}(a) b .
$$

We have $i_{1}=x, j_{1}=\operatorname{res}_{G, N}$, and $k_{1}=\operatorname{Tr}_{N, G}$ so that the differential $d_{1}$ is equal to $1+g=\operatorname{res}_{G, N} \circ \operatorname{Tr}_{N, G}$. By the usual identification of the differentials in the spectral sequence (see for example, [2, pp. 103-105]), an element $x^{p} \otimes y$ survives to represent an element $\left[x^{p} \otimes y\right]$ in $E_{n}^{p q}$ if and only if there is an element $z \in H^{q-n+1}(G, k)$ with $\operatorname{Tr}_{N, G}(y)=x^{n-1} z$, and then we have

$$
d_{n}\left[x^{p} \otimes y\right]=\left[x^{p+n} \otimes \operatorname{res}_{G, N}(z)\right] \in E_{n}^{p+n, q-n+1} .
$$


Unfiltering, it follows that $d_{n} \neq 0$ if and only if there is an element $z \in$ $H^{*}(G, k)$ with $x^{n} z=0$ but such that there is no element $u \in H^{*}(G, k)$ with $x^{n-1} z=x^{n} u$. This is because if the latter holds then according to the above long exact sequence $x^{n-1} z$ is in the image of transfer. If $x^{n-1} z=\operatorname{Tr}_{N, G}(y)$ then $d_{n}[1 \otimes y]=\left[x^{n} \otimes z\right] \neq 0$ in $E_{n}^{n, *}$.

\section{THE EXAMPLE}

According to the analysis of the last section, in order to produce an example of a split extension

$$
1 \rightarrow N \rightarrow G \rightarrow \mathbf{Z} / 2 \rightarrow 1
$$

with $d_{n} \neq 0$ in the corresponding spectral sequence, it suffices to produce a group $G$ with elements $x \in H^{1}\left(G, \mathrm{~F}_{2}\right)$ and $z \in H^{*}(G, k)$ with $x$ nonnilpotent and $x^{n} z=0$ but $x^{n-1} z \neq 0$. We take for $G$ a nilpotent group of class two of the form

$$
1 \rightarrow(\mathbf{Z} / 2)^{n} \rightarrow G \rightarrow(\mathbf{Z} / 2)^{n+1} \rightarrow 1 .
$$

The center $Z(G)$ is an elementary abelian group of order $2^{n}$ generated by commuting involutions $h_{1}, \ldots, h_{n}$. The group $G$ is generated by these (redundant) generators together with generators $g_{0}, \ldots, g_{n}$ commuting with $h_{1}, \ldots, h_{n}$ and satisfying $g_{0}^{2}=1, g_{1}^{2}=1, g_{j}^{2}=h_{j}(j \geq 2),\left[g_{0}, g_{1}\right]=h_{1},\left[g_{j}, g_{j+1}\right]=$ $h_{j}(j \geq 1)$, and the remaining commutators $\left[g_{i}, g_{j}\right]$ are equal to 1 .

We have $H^{*}(G / Z(G), k)=k\left[x_{0}, \ldots, x_{n}\right], H^{*}(Z(G), k)=k\left[y_{1}, \ldots, y_{n}\right]$, where the $x$ 's and $y$ 's are the dual bases to the $g$ 's and $h$ 's in $H^{1}(G / Z(G), k)$ $\cong \operatorname{Hom}(G / Z(G), k)$ and $H^{1}(Z(G), k) \cong \operatorname{Hom}(Z(G), k)$, respectively.

In the spectral sequence

$$
H^{*}\left(G / Z(G), H^{*}(Z(G), k)\right) \Rightarrow H^{*}(G, k)
$$

we have

$$
\begin{aligned}
E_{2}^{* *} & =k\left[x_{0}, \ldots, x_{n}\right] \otimes k\left[y_{1}, \ldots, y_{n}\right], \\
d_{2}\left(y_{1}\right) & =x_{0} x_{1}+x_{1} x_{2}, \\
d_{2}\left(y_{j}\right) & =x_{j}^{2}+x_{j} x_{j+1} \quad(2 \leq j \leq n-1), \\
d_{2}\left(y_{n}\right) & =x_{n}^{2} .
\end{aligned}
$$

It is easy to check using Macaulay's criterion (Zariski and Samuel [5, Appendix 6 , Theorem 2]) that $d_{2}\left(y_{1}\right), \ldots, d_{2}\left(y_{n}\right)$ form a regular sequence in $k\left[x_{0}, \ldots, x_{n}\right]$, so

$$
E_{3}^{* *}=k\left[x_{0}, \ldots, x_{n}\right] /\left(d_{2}\left(y_{1}\right), \ldots, d_{2}\left(y_{n}\right)\right) \otimes k\left[y_{1}^{2}, \ldots, y_{n}^{2}\right] .
$$

Now, for each $j(1 \leq j \leq n), d_{3}\left(y_{j}^{2}\right)=\mathrm{Sq}^{1} d_{2}\left(y_{j}\right)$ is in the ideal generated by $d_{2}\left(y_{j}\right)$, so $d_{3}=0$. It follows that all further differentials are zero, and there are no ungrading problems, so $H^{*}(G, k) \cong E_{\infty} \cong E_{3}$.

The Poincare series of the ring $k\left[x_{0}, \ldots, x_{n}\right] /\left(d_{2}\left(y_{1}\right), \ldots, d_{2}\left(y_{n}\right)\right)$ is $\left(1-t^{2}\right)^{n} /(1-t)^{n+1}=(1+t)^{n} /(1-t)$. The relations allow every element to be expressed as a linear combination of monomials $x_{0}^{\alpha_{0}} x_{1}^{\alpha_{1}} \cdots x_{n}^{\alpha_{n}}$ with either $\alpha_{0}=0$ or $\alpha_{1}=0$ and each $\alpha_{j}=0$ or 1 for $2 \leq j \leq n$. The Poincaré series then forces these elements to be linearly independent. So

$$
x_{0}^{n} x_{1}=x_{1} x_{2}^{n}=x_{1} x_{2} x_{3}^{n-1}=\cdots=x_{1} x_{2} \cdots x_{n}^{2}=0,
$$


while

$$
x_{0}^{n-1} x_{1}=x_{1} x_{2}^{n-1}=x_{1} x_{2} x_{3}^{n-2}=\cdots=x_{1} x_{2} \cdots x_{n} \neq 0
$$

is an element which is linearly independent of $x_{0}^{n}$ and hence not divisible by $x_{0}^{n}$. So we may take $x$ to be $x_{0}$ and $z$ to be $x_{1}$. In other words, $N$ is the normal subgroup of index two generated by $g_{1}, \ldots, g_{n}, h_{1}, \ldots, h_{n}$, and $x_{0}^{n} \otimes x_{1}$ is in the image of $d_{n}$ in the spectral sequence

$$
H^{*}\left(G / N, H^{*}(N, k)\right) \Rightarrow H^{*}(G, k) \text {. }
$$

\section{REFERENCES}

1. G. Avrunin and J. F. Carlson, Nilpotency degree of cohomology rings in characteristic two, Proc. Amer. Math. Soc. 118 (1993), 339-343.

2. D. J. Benson, Representations and cohomology, II. Cohomology of groups and modules, Cambridge Stud. Adv. Math., vol. 31, Cambridge Univ. Press, Cambridge and New York, 1991.

3. M. Feshbach, The image of $H^{*}(B G ; \mathbf{Z})$ in $H^{*}(B T ; Z)$ for $G$ a compact Lie group with maximal torus $T$, Topology 20 (1981), 93-95.

4. D. Quillen, The spectrum of an equivariant cohomology ring. I, II, Ann. of Math. (2) 94 (1971), 549-602.

5. O. Zariski and P. Sammuel, Commutative algebra, Vol. II, Graduate Texts in Math., vol. 29, Springer-Verlag, Berlin and New York, 1975.

Mathematical Institute, 24-29 St. Giles, Oxford OX1 3LB, Great Britain

Current address: D. J. Benson: Department of Mathematics, University of Georgia, Athens, Georgia 30602

E-mail address: DJB@SLOTH.MATH.UGA.EDU

Department of Mathematics, University of Minnesota, Minneapolis, Minnesota 55455 